\title{
DIVERGENT PATTERNS OF INCORPORATION OF BROMODEOXYURIDINE AND IODODEOXYURIDINE IN HUMAN COLORECTAL TUMOR CELL LINES
}

\author{
Jonathan Maybaum,${ }^{*} \dagger$ Eric C. Burton,$\dagger$ David A. Shelton,$\dagger$ Hong-Wei Jing,$\dagger$ \\ Christine E. Dusenbury, $\dagger$ William D. Ensminger $\ddagger$ and Philip L. STetson $\dagger$ \\ Departments of $\dagger$ Pharmacology and $\ddagger$ Internal Medicine, Upjohn Center for Clinical Pharmacology, \\ University of Michigan Medical School, Ann Arbor, MI 48109, U.S.A.
}

(Received 17 November 1990; accepted 28 January 1991)

\begin{abstract}
Using a panel of four human colorectal tumor (HCT) cell lines, we have quantitatively characterized the incorporation of bromodeoxyuridine (BrdUrd) and iododeoxyuridine (IdUrd) into DNA, both as individual agents and in combination with fluoropyrimidines. The intrinsic ability of these cell lines to incorporate BrdUrd, as reflected by the concentration required to achieve halfmaximal incorporation, varied almost 4-fold across this panel, from $1.6 \mu \mathrm{M}$ for HuTu80 cells to $6.1 \mu \mathrm{M}$ for HT29 cells. Three of the four cell lines (HT29, SW480, SW620) responded to fluoropyrimidines as expected, displaying 100-150\% increases in BrdUrd incorporation when combined with growth inhibitory concentrations of fluorouracil (FUra). In contrast, neither FUra nor fluorodeoxyuridine (FdUrd) was able to increase BrdUrd incorporation in HuTu80 cells by more than $25 \%$, even in the presence of $100 \mu \mathrm{M}$ leucovorin. IdUrd incorporation was modulated to a substantially higher degree in both HT29 and HuTu80 cell lines. Finally we demonstrate the feasibility of a technique for evaluating the net effect of fluoropyrimidine treatments on de novo thymidine nucleotide production in a single specimen, using a combination of normotopic and stable-isotope labeled BrdUrd. We propose that this approach may be useful in evaluating the response of an individual tumor to fluoropyrimidines in vivo.
\end{abstract}

Tumors of colorectal origin are among the most common to afflict the general adult population. While treatment of this type of tumor has become increasingly effective, employing combinations of surgery, radiation and chemotherapy (chiefly antimetabolites), a significant portion of these tumors respond poorly to therapeutic treatments. Although no single determinant of clinical responsiveness has been identified as a result of these investigations, it has become evident that a great deal of heterogeneity exists among human colorectal tumors with regard to both their metabolism of fluoropyrimidines and their apparent sensitivity to the major biochemical lesions induced by these agents [i.e. inhibition of thymidine (dThd§) nucleotide synthesis and analog incorporation into RNA and DNA] [see, for example, Refs. 1 and 2].

Our efforts to improve the response rate of colorectal tumors have recently concerned the use of other halopyrimidines, bromodeoxyuridine (BrdUrd) and iododeoxyuridine (IdUrd), whose incorporation into cellular DNA is known to sensitize cells to $\mathrm{UV}$ and $\mathrm{X}$-irradiation [3]. Since these analogs compete with dThd for incorporation into DNA,

\footnotetext{
* Corresponding author: Jonathan Maybaum, Ph.D. 4302A Upjohn Center, University of Michigan Medical School, Ann Arbor, MI 48109-0504.

§ Abbreviations: BrdUrd, bromodeoxyuridine; BrdUMP, bromodeoxyuridine monophosphate; dThd, thymidine; FdUrd, fluorodeoxyuridine; FUra, fluorouracil; HCT, human colorectal tumor; IdUrd, iododeoxyuridine; IdUMP, iododeoxyuridine monophosphate; and TS, thymidylate synthase.
}

it is predictable that inhibition of endogenous thymidylate synthesis by fluorouracil (FUra) or fluorodeoxyuridine (FdUrd) should enhance their incorporation and, therefore, their efficacy as radiosensitizers. Based on this interaction, Phase I/ II clinical trials are in progress in which either BrdUrd or IdUrd is combined with FUra for the purpose of potentiating incorporation of the thymidine analogs. However, in light of the abovementioned heterogeneity, it would be expected that the effectiveness of modulating BrdUrd or IdUrd incorporation with fluoropyrimidines may vary considerably among human colorectal tumor cell lines. Since addition of a fluoropyrimidine to a BrdUrd or IdUrd regimen potentially poses an increased hazard, it would be useful to quantitatively characterize the benefit derived (i.e. increased incorporation) under conditions approximating those expected to be operative in vivo. Therefore, we have performed the present study, using human colorectal tumor cell lines which have been shown previously to have widely divergent responses to fluoropyrimidines [4-6], to assess the relationship between intrinsic ability to incorporate BrdUrd into DNA and the modulation of this process by fluoropyrimidines.

\section{MATERIALS AND METHODS}

Cell culture and drug treatments. All cell lines were purchased from the American Type Culture Collection and maintained at $37^{\circ}$ in a $5 \% \quad \mathrm{CO}_{2}$ atmosphere, in McCoy's 5A medium supplemented with $10 \%$ fetal bovine serum (both from GIBCO). Undialyzed serum was used except where indicated. 
Table 1. Halopyrimidine incorporation parameters for human colorectal tumor cell lines

\begin{tabular}{|c|c|c|c|c|c|c|}
\hline \multirow[b]{2}{*}{ Cell line } & \multicolumn{3}{|c|}{ BrdUrd } & \multicolumn{3}{|c|}{ IdUrd } \\
\hline & $\mathrm{I}_{\max }(\%)$ & $C_{50}(\mu \mathrm{M})$ & $C_{10}(\mu \mathrm{M})$ & $I_{\max }(\%)$ & $C_{50}(\mu \mathrm{M})$ & $C_{10}(\mu \mathrm{M})$ \\
\hline HuTu80 & $36.4 \pm 1.3(4)$ & $1.6 \pm 0.2(4)$ & 0.18 & $37.2(2)$ & $7.5(2)$ & 0.8 \\
\hline SW480 & $50.7 \pm 0.8$ & $3.6 \pm 0.9$ & 0.39 & & & \\
\hline SW620 & $47.8 \pm 0.8(3)$ & $4.6 \pm 0.3(3)$ & 0.51 & & & \\
\hline HT29 & $48.0 \pm 1.2(5)$ & $6.1 \pm 0.9(5)$ & 0.68 & $49.8 \pm 0.4(3)$ & $27.1 \pm 0.7$ & 3.0 \\
\hline
\end{tabular}

Cells were exposed to a series of BrdUrd/IdUrd concentrations for one doubling time after which the extent of replacement of dThd was measured by GC/MS. Data were fitted to the equation: $I=$ $\left(C \cdot I_{\max }\right) /\left(C+I_{\max }\right)$, as described previously [9]. From the resulting fit we calculated the concentrations at which 10 or $50 \%$ of maximal incorporation would be expected $\left(C_{10}\right.$ or $\left.C_{50}\right)$, and the percent replacement was projected at infinitely high BrdUrd or IdUrd concentration $\left(I_{\max }\right)$. Values are means $\pm \mathrm{SEM}(\mathrm{N})$ or an average of two determinations. The $C_{10}$ values were calculated from the mean values for $I_{\max }$ and $C_{50}$.

The doubling time for HT29, SW480 and SW620 cells was typically $24 \mathrm{hr}$, while for HuTu80 cells it was $16 \mathrm{hr}$. Cultures were screened for mycoplasma contamination approximately every 3 months.

Stable-isotope labeled BrdUrd $\left[1,3,-{ }^{15} \mathrm{~N}, 2-{ }^{13} \mathrm{C}\right]$, $\geqslant 99 \%$ pure by mass spectrometric analysis, was custom synthesized by MSD Isotopes, Montreal, Canada. All other drugs and reagents were purchased from Sigma Biochemicals. For incorporation experiments, cells were plated at $20,000 / \mathrm{cm}^{2}$ and allowed to recover for two doubling times before treatment.

Analysis of BrdUrd and IdUrd incorporation. These determinations were performed as described previously $[7,8]$. Briefly, cells were lysed in a buffer containing sodium dodecyl sulfate, followed by proteinase and RNase digestion and precipitation of cellular DNA with ethanol. Enzymatic hydrolysis with DNase I, phosphodiesterase, alkaline phosphatase and thymidine phosphorylase liberated incorporated 5-bromouracil and thymine. After addition of 5-chlorouracil as an internal standard, the bases were then extracted into ethyl acetate and derivatized with bis-trimethylsilyl trifluoroacetamide. Quantitation of the derivatized products was carried out using a Hewlett-Packard 5987A gas chromatograph/mass spectrometer in selected ion monitoring mode. Initial experiments showed that standard curves for isotopic and normotopic BrdUrd were indistinguishable; therefore, a standard curve for only normotopic BrdUrd was constructed and used for both BrdUrd species.

Measurement of growth inhibition. Cells were seeded in 24-well plates at a density of $20,000 / \mathrm{cm}^{2}$ (except for HuTu80, $7500 / \mathrm{cm}^{2}$ ) and were allowed to recover for 2 days, after which their medium was replaced with medium containing FdUrd. After approximately 3-4 doubling times, cells were harvested and counted using a Coulter Counter. Cell growth was calculated by subtracting the number of cells present at the beginning of drug treatment from the final number. All treatments were performed in triplicate, using dialyzed serum.

\section{RESULTS}

Quantitative analysis of BrdUrd/IdUrd incorporation without modulation. Previous studies from

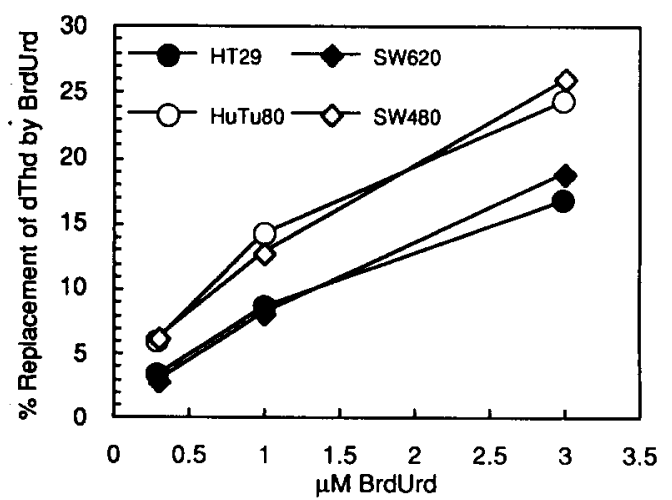

Fig. 1. Incorporation in HCT cell lines at low BrdUrd concentrations. Cells were plated at $20,000 / \mathrm{cm}^{2}$, allowed to recover for two doubling times, and then exposed to the indicated concentrations of BrdUrd for one doubling time. Incorporation of BrdUrd was measured by $\mathrm{GC} / \mathrm{MS}$ analysis and the data presented are mean values \pm SEM for triplicate samples from a single, representative experiment.

our group have indicated that the quantitative relationship between external concentrations of halogenated dThd analogs and the consequent extent of their incorporation into DNA in place of dThd can be closely fit to a Langmuir equation [9]. The parameters derived from such a fit $\left(C_{50}\right.$ and $\left.I_{\max }\right)$ are indicative of the concentration-dependence and the maximum possible efficiency, respectively, of the overall incorporation process. Table 1 gives values observed for these parameters in four HCT cell lines for BrdUrd and two lines for IdUrd; in addition, values were calculated for the concentration expected to give $10 \%$ maximal incorporation in each instance $\left(C_{10}\right)$. Also, the actual values for incorporation at low BrdUrd concentrations are shown in Fig. 1. While the values for incorporation for these cell lines tend to converge at $10 \mu \mathrm{M}$ and above (not shown), these data demonstrate that in the clinically relevant (low micromolar) range, the intrinsic ability of HCT cell lines to incorporate BrdUrd is quite variable.

Modulation of BrdUrd/IdUrd incorporation by 


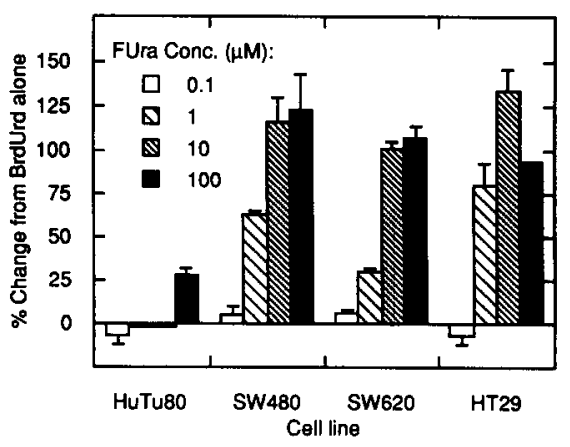

Fig. 2. Modulation of BrdUrd incorporation by FUra in a panel of HCT cell lines. Cells were exposed to the $C_{10}$ concentration of BrdUrd (see Table 1) plus the indicated concentration of FUra for one doubling time. The results are expressed as percent change of each FUra-treated sample compared to the mean incorporation of the samples exposed to BrdUrd only. The data presented are mean values \pm SEM for triplicate samples from a single, representative experiment. Control values for percent replacement by BrdUrd in these experiments were: HuTu80, 4.7; SW480, 3.4; SW620, 3.3; and HT29, 6.2.

FUra/FdUrd. Our initial assessment of modulation was performed by treating cells with the $C_{10}$ concentration of BrdUrd for one doubling time, in combination with various FUra concentrations (Fig. 2). Although the qualitative result that FUra generally enhanced BrdUrd incorporation was expected, the quantitative profile of this effect was puzzling in some respects. Several aspects of the response of HT29 and HuTu80 cells to fluoropyrimidines were characterized previously by Washtien $[4,5]$ and, based on these results, we had predicted that modulation of BrdUrd incorporation should be significant in the HT2 2 cells at about $1 \mu \mathrm{M}$ FUra and in the HuTu80 cells at about $10 \mu \mathrm{M}$ FUra. While the HT29 cells responded as expected, BrdUrd incorporation in the HuTu80 cells was not affected by $10 \mu \mathrm{M}$ FUra, even though this concentration reportedly was sufficient to titrate all of the thymidylate synthase (TS) in this line [4]. Also, the finding that $100 \mu \mathrm{M}$ FUra produced less modulation than $10 \mu \mathrm{M}$ FUra in HT29 cells was initially unexpected. This result, which was reproducible obtained in several experiments, may be due to growth inhibition caused by FUra incorporation into RNA.

To further characterize the differences in modulation between HT29 and HuTu80 cells, we next determined the dependence of modulation on BrdUrd concentration. As illustrated in Fig. 3, relative increases in BrdUrd incorporation observed using the $C_{50}$ concentration for BrdUrd were similar to those seen at the $C_{10}$ concentration in each cell line. This was the case when either $100 \mu \mathrm{M}$ FUra or $100 \mathrm{nM}$ FdUrd was used as the modulating agent. Since it is now well known that reduced folate cofactors are, in some cases, the limiting factor in determining the extent of TS inhibition by fluorodeoxyuridine monophosphate, we repeated the experiments shown in Fig. 3 with the addition
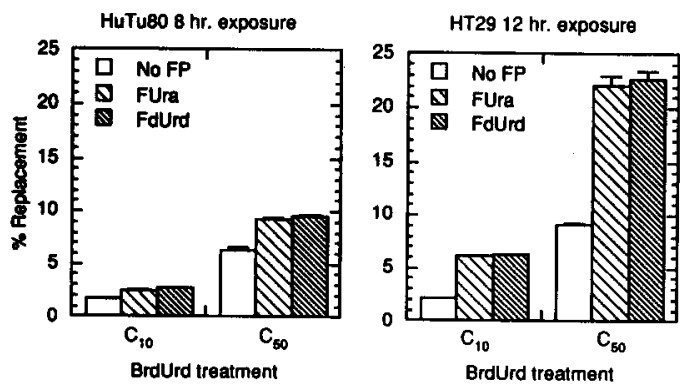

Fig. 3. Effect of BrdUrd concentration on modulation by FUra or FdUrd. HT29 or HuTu80 cells were exposed to either the $C_{10}$ or $C_{50}$ BrdUrd concentration plus $100 \mu \mathrm{M}$ FUra or $100 \mathrm{nM}$ FdUrd (or no fluoropyrimidine) for onehalf of a doubling time. Values are means \pm SEM for the percent replacement of dThd by BrdUrd (i.e. BrdUrd incorporation). These data are from a single, representative experiment (using dialyzed serum) in which triplicate samples were processed.

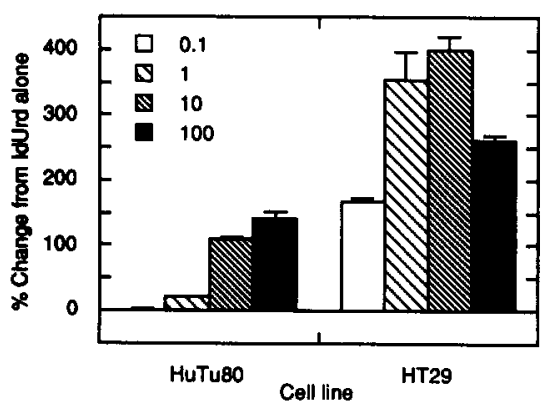

Fig. 4. Modulation of IdUrd incorporation by FUra Modulation of IdUrd incorporation by FUra in HT29 and HuTu80 cells was measured as in Fig. 2 for BrdUrd. Control values for percent replacement by IdUrd in these experiments were: HuTu80, 4.4; and HT29, 4.5. Values are means $\pm S E M$ for triplicate samples from a single representative experiment.

of $100 \mu \mathrm{M}$ leucovorin. The values observed for incorporation were virtually identical in the absence or presence of leucovorin, (data not shown).

Modulation of IdUrd incorporation by FUra was studied in HT29 and HuTu80 cells, at the $C_{10}$ concentrations defined in Table 1. In both cell lines, IdUrd incorporation was enhanced to a substantially higher degree than that of BrdUrd (Fig. 4), although the effect in the HuTu80 line was still much less than in HT29 cells. Perhaps the most striking difference between the two analogs is the contrast in HuTu80 cells of the effect of $10 \mu \mathrm{M}$ FUra/BrdUrd, which produced no modulation, compared to $10 \mu \mathrm{M}$ FUra/IdUrd, where the fluoropyrimidine doubled analog incorporation.

Growth inhibition by FdUrd. Although FdUrdinduced growth inhibition for three of the lines used here was studied by Washtien [5], the modulation patterns we observed induced us to reexamine this effect. While the responses of the HT29 and SW480 cells in our hands were virtually identical to those 


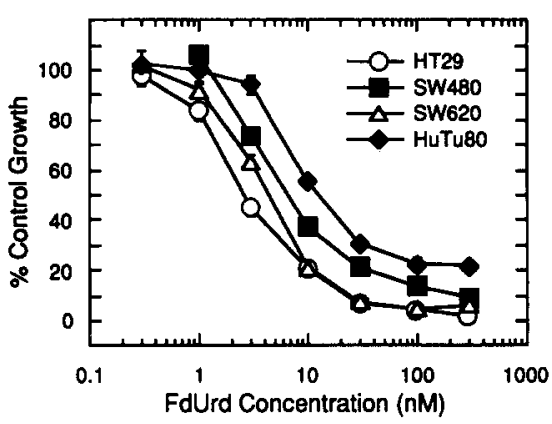

Fig. 5. Growth inhibition of HCT cell lines by FdUrd. Cells were plated onto 24-well dishes and allowed to recover for 2 days, at which time their medium was replaced with FdUrd-containing medium. Growth inhibition was determined after 3-4 doubling times. Values are the means \pm SEM for three separate experiments.

reported earlier (Fig. 5), the HuTu80 line was slightly different in that, even at $300 \mathrm{nM}$ FdUrd these cells grew at $20 \%$ of control rate.

Measurement of modulation using stable-isotope labeled BrdUrd. Although measurement of modulation by comparing separate flasks of cells processed in parallel (with or without modulator) is the most direct experimental approach in vitro, this scheme would be impractical in vivo, where it is likely that only a single sample would be available from each treated subject. One way of circumventing this problem is to design the treatment protocol so that BrdUrd incorporated before addition of the modulator can be measured separately from that incorporated in the presence of the modulator, in the same sample. Since our technique for measuring BrdUrd incorporation employs mass spectrometric detection, such a protocol is possible by using stableisotope labeled BrdUrd.

Figure 6 gives the results of an experiment in which HuTu80 and HT29 cells were exposed to $1 \mu \mathrm{M}$ normotopic BrdUrd for one-sixth of a doubling time ( 3 or $4 \mathrm{hr}$, respectively), at which point the cells were washed and incubated for the same time period with $1 \mu \mathrm{M}$ isotopic BrdUrd + the indicated concentration of FUra. As expected, the ratio of isotopic/normotopic BrdUrd was approximately doubled in HT29 cells, compared to controls, when concentrations of FUra were $1 \mu \mathrm{M}$ or higher. In HuTu80 cells this ratio was increased slightly at $100 \mu \mathrm{M}$ FUra and essentially unchanged at lower FUra concentrations. The pattern of response to FdUrd was similar in that, in HT29 cells, modulation paralleled growth inhibition, whereas HuTu80 cells were essentially resistant to modulation even at high FdUrd concentrations. For both cell lines the absolute degree of modulation with FdUrd was slightly higher than with FUra. We also observed that the order of administration of the isotopic and normotopic forms of the drug made no detectable difference in the results obtained.

\section{DISCUSSION}

The finding that BrdUrd incorporation could not
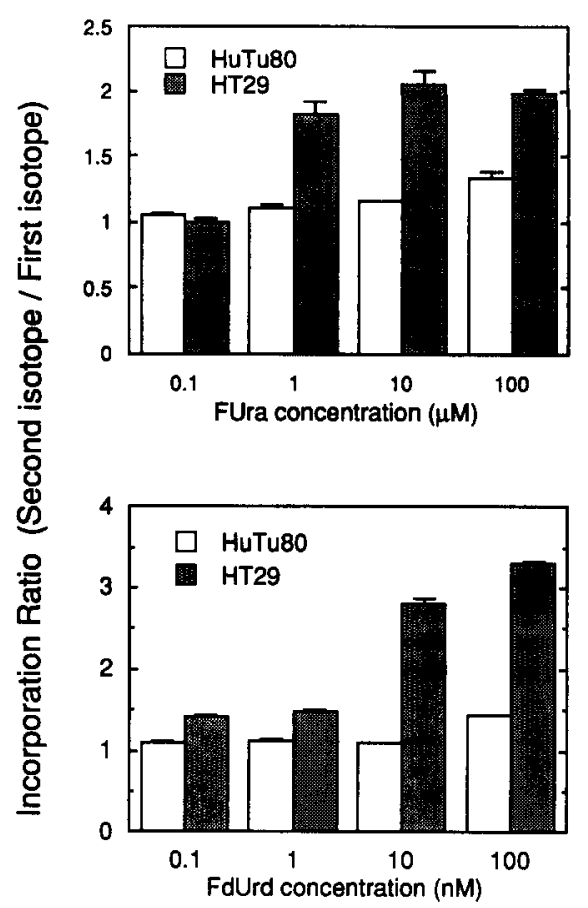

Fig. 6. Measurement of modulation using stable-isotope labeled BrdUrd. HuTu80 or HT29 cells were exposed to $1 \mu \mathrm{M}$ normotopic BrdUrd for one-sixth of a doubling time ( 3 or $4 \mathrm{hr}$, respectively), at which point the cells were washed and incubated for the same time period with $1 \mu \mathrm{M}$ isotopic BrdUrd + the indicated concentration of FUra. Values are the means \pm SEM for three independent samples from a single, representative experiment.

be enhanced substantially in HuTu80 cells, even by high fluoropyrimidine concentrations, was the most surprising result of this study. We had originally expected that modulation would be closely correlated with growth inhibition, but even though $10 \mathrm{nM}$ FdUrd/HT29 and $100 \mathrm{nM}$ FdUrd/HuTu80 both represent treatments which inhibit growth by $80 \%$, incorporation of BrdUrd was almost tripled in HT29 cells while it was increased by less than $50 \%$ in HuTu80 cells, compared to cells exposed to BrdUrd alone. Furthermore, while $10 \mathrm{nM}$ FdUrd caused about $50 \%$ growth inhibition in HuTu80 cells, it did not enhance BrdUrd incorporation at all. We were initially reluctant to conclude that such growth inhibitory treatments were truly ineffective at modulating BrdUrd utilization in HuTu80 cells, and we therefore considered some potential artifactual bases for these observations.

Since BrdUrd can be depleted rapidly from medium $[10,11]$ and since the initial concentration of BrdUrd in the experiments shown in Fig. 2 was quite low $(0.2 \mu \mathrm{M})$, we hypothesized that the lack of modulation might be explained by complete utilization of the extracellular BrdUrd. In that case all of the available BrdUrd would have been used without modulator present, providing no advantage upon the addition of modulator. However, measurement of BrdUrd levels in medium incubated with HuTu80 cells showed minimal disappearance of the 
drug, even over a 24-hr time period (not shown), indicating that depletion of BrdUrd is not the basis for lack of modulation.

Another possible artifact stems from the use of undialyzed serum in our initial experiments. We determined that the level of dThd in our medium was typically 0.1 to $0.2 \mu \mathrm{M}$, which is of the same order of magnitude as the $C_{10}$ for BrdUrd in HuTu80 cells. One could postulate that if this line were especially proficient at utilizing exogenous dThd (as is suggested by efficient incorporation of BrdUrd at low concentrations), then the extent of BrdUrd replacement might be more dependent on the exogenous BrdUrd:dThd ratio than on the contribution of de novo dThd nucleotides. The data in Fig. 3 argue against this interpretation, though, since they show that use of dialyzed serum and of a higher BrdUrd concentration $(1 \mu \mathrm{M})$ did not increase modulation. Therefore, we have accepted the conclusion that the refractoriness of HuTu80 cells to fluoropyrimidine modulation is a function of the physiology of that cell line, rather than an experimental anomaly.

One possible explanation for the failure of moderate FdUrd concentrations to modulate BrdUrd incorporation is by an alteration of the intracellular disposition (specifically the catabolism) of BrdUrd, by FdUrd treatment. As mentioned earlier, BrdUrd depletion from the medium by HuTu80 cells was negligible. In contrast, incubation of BrdUrdcontaining medium with HT29 cells resulted in extensive depletion of this nucleoside from the medium [11], accompanied by appearance in the medium of the corresponding base, bromouracil (data not shown). These data indicate that, while intracellular breakdown of BrdUrd by dThd phosphorylase is significant in HT29 cells, this process is minimal in HuTu80 cells, indicating that in the latter line dThd phosphorylase is either absent, inactive or saturated with endogenously produced dThd. If the failure of BrdUrd to be catabolized extensively is due to saturation of phosphorylase, then reduction of endogenous dThd synthesis could free some of this activity to degrade BrdUrd. It this scheme is correct, then one would expect to find substantial amounts of thymine in medium incubated with HuTu80 cells and it would also be predicted that FdUrd treatment should increase the conversion of BrdUrd to BrUra in this line.

A second possibility is that the refractoriness of HuTu80 cells to modulation at high FP concentrations could be due to inhomogeneity of TS in this line. Since FUra and FdUrd are equally ineffective modulators of BrdUrd in HuTu80 cells, it seems likely that inhibition of the common target enzyme for these drugs, TS, is the critical determinant rather than, for example, anabolism of either drug. However, total cellular content of TS appears not to be predictive of modulation since SW480 cells have about five times as much TS as HT29 cells, while the concentration-dependence of FUrainduced modulation is similar in these two lines [5]. Furthermore, TS concent of HuTu80 cells is only about twice that of the SW480 line and yet there is at least a 100 -fold difference in the concentration of
FUra needed to achieve the same degree of modulation in the two lines.

While the bulk of HuTu80 TS seems to be inhibitable at concentrations only slightly higher than the other three cell lines, as suggested by the growth inhibition curves in Fig. 5, the failure of much higher concentrations of FdUrd to depress HuTu80 growth below $20 \%$ of control suggests that a subset of the TS of that cell line is refractory to inhibition. These findings are consistent with the concept that several of the enzymes involved in producing DNA precursors, including TS, are physically compartmentalized $[12-14]$, and with some recent studies which specifically point to heterogeneity of intracellular TS activities $[15,16]$. Alternatively, HuTu 80 cells may contain both wild type and variant forms of TS, as has been demonstrated in the HCT116 line $[17,18]$. Both of these cases would predict the existence of a vulnerable subclass of TS which would bear the brunt of fluoropyrimidine treatment, and a privileged subclass of TS whose continued activity accounts for poor modulation of BrdUrd incorporation.

Preferential inhibition of a vulnerable subclass of TS could also provide an explanation for the differences between modulation of BrdUrd and IdUrd in HuTu80 cells, if dehalogenation of IdUMP is significant in this line. Conversion of IdUMP to dUMP by TS has been demonstrated previously in a cell-free system [19] and in a number of cell lines $[20,21]$. BrdUMP can also be dehalogenated, with an efficiency somewhat less than IdUMP, depending on the conditions used [19]. In both the HT29 and HuTu80 cell lines, modulation of IdUrd incorporation was significantly higher than that of BrdUrd. Although vulnerable TS may not contribute substantially to replicative DNA synthesis in $\mathrm{HuTu} 80$ cells, its dehalogenation of BrdUMP and IdUMP could be significant. By this reasoning we would expect that modulation of IdUrd in HuTu80 cells is due almost exclusively to reduced dehalogenation by vulnerable TS, while in HT29 cells modulation is due to a combination of less dehalogenation and less production of de novo thymidylate.

Since it has been shown that dThd and its analogs can alter uptake of FdUrd by affecting dThd kinase activity [22], it is possible that co-incubation of BrdUrd with FdUrd may attenuate activation of the latter by dThd kinase. As indicated, earlier, however, the fact that both FdUrd and FUra are both poor modulators at high concentrations in HuTu80 cells argues against an important role for anabolism in this system.

Although the correct explanation for our observations is not defined as yet, one thing that is clear from the data presented here is that there is likely to be a considerable degree of variability among human colorectal tumors with respect to both intrinsic ability to incorporate BrdUrd and ability to modulate that incorporation by FUra or FdUrd. Furthermore, in view of the complexity of the models needed to rationalize our observations in vitro, prediction of the incorporation or modulation properties of a tumor in vivo will probably not be successful if based on measurements of individual pools or enzyme activities. While measurement of 
incorporation with and without modulator is the most direct approach to characterizing the properties of a tumor in a given individual, one would need to take separate biopsy samples both before and after adding modulator to a BrdUrd infusion. In addition to the difficulties involved in obtaining serial specimens, normalization of incorporation values between the two samples would be quite problematic.

In anticipation of this potential problem we have used the unique capability of our GC/MS assay to distinguish stable-isotope labeled BrdUrd, incorporated in the absence of modulator, from normotopic BrdUrd, incorporated in the presence of the modulator. This approach permits assessment of modulation by a selected fluoropyrimidine treatment in a single specimen which would, in effect, act as its own control. As shown in Fig. 6, the double-label protocol gives results equivalent to measurement of modulation by comparison of samples processed in parallel. If both BrdUrd and IdUrd were used simultaneously, it might be possible to additionally obtain information about the extent of dehalogenation in a sample, by comparing the modulation of the two dThd analogs. Finally, if we are at some point able to satisfactorily reconcile the effects of fluoropyrimidines on modulation and growth inhibition, measurement of modulation using stable isotopes may be useful to predict the therapeutic efficacy of fluoropyrimidine treatments intended to be cytotoxic, rather than modulatory.

In summary, we have shown that HCT cell lines vary considerably with regard to both their intrinsic ability to incorporate halogenated dThd analogs, as well as the effectiveness of fluoropyrimidines to modulate this incorporation. We speculate that the lack of an obvious relationship between reported TS content, growth inhibition and susceptibility to modulation may be due to differences among these lines in the intracellular distribution of this enzyme, or to the existence of a variant form of TS in the $\mathrm{HuTu} 80$ line. We propose that analysis of biopsy specimens following treatment with normotopic and stable-isotope labeled BrdUrd (and perhaps IdUrd) could be used to evaluate the sensitivity of individual tumors to modulation of analog incorporation by fluoropyrimidine treatment, and that this information would be useful not only for improving the use of these dThd analogs as radiosensitizers, but eventually for also predicting intrinsic sensitivity of individual tumors to fluoropyrimidine treatment.

Acknowledgements - This work was supported by $\mathrm{NIH}$ Grants CA42761 and CA46256.

\section{REFERENCES}

1. Houghton JA, Maroda SJ Jr, Phillips JO and Houghton PJ, Biochemical determinants of responsiveness to 5fluorouracil and its derivatives in xenografts of human colorectal adenocarcinomas in mice. Cancer Res 41: 144-149, 1981.

2. Spears CP, Gustavsson BG, Berne M, Frösing R,
Bernstein L and Hayes AA, Mechanisms of innate resistance to thymidylate synthase inhibition after 5fluorouracil. Cancer Res 48: 5894-5900, 1988.

3. Szyblaski W, X-Ray sensitization by halopyrimidines. Cancer Chemother Rep 58: 539-557, 1974.

4. Washtien WL, Comparison of 5-fluorouracil metabolism in two gastrointestinal tumor cell lines. Cancer Res 44 909-914, 1984

5. Washtien WL, Thymidylate synthetase levels as a factor in 5-fluorodeoxyuridine and methotrexate cytotoxicity in gastrointestinal tumor cells. Mol Pharmacol 21: 723$728,1982$.

6. Drewinko B and Yang LY, Cellular basis for the inefficacy of 5-FU in human colon carcinoma. Cancer Treat Rep 69: 1391-1398, 1985.

7. Maybaum J, Kott MG, Johnson NJ, Ensminger WD and Stetson PL, Analysis of bromodeoxyuridine incorporation into DNA: Comparison of gas chromatographic/mass spectrometric, $\mathrm{CsCl}$ gradient sedimentation, and specific radioactivity methods. Anal Biochem 161: 164-171, 1987.

8. Stetson PL, Maybaum J, Shukla UA and Ensminger WD, Simultaneous determination of thymine and 5bromouracil in DNA hydrolysates using gas chromatography-mass spectrometry with selected-ion monitoring. J Chromatogr 375: 1-9, 1986.

9. Stetson PL, Maybaum J, Wagner JG, Averill DR, Wollner IS, Knol JA, Johnson NJ, Yang Z, Preiskorn D, Smith P, Knutsen CA and Ensminger WD, Tissuespecific pharmacodynamics of 5-bromo-2'-deoxyuridine incorporation into DNA in VX2 tumor-bearing rabbits. Cancer Res 48: 6900-6905, 1988.

10. Wolff $\mathrm{S}$ and Fijtman $\mathrm{N}$, X-Ray sensitization of chromatids with unifilarly and bifilarly substituted DNA. Mutat Res 80: 133-140, 1981.

11. Maybaum J, Hafner MS, Burton EC, Stetson PL, Ensminger WD and Rogers CE, Response of human HT-29 colorectal tumor cells to extended exposure to bromodeoxyuridine. Cancer Chemother Pharmacol 25: 45-50, 1989.

12. Prem veer Reddy $G$ and Pardee AB, Multienzyme complex for metabolic channeling in mammalian DNA replication. Proc Natl Acad Sci USA 77: 3312-3316, 1980.

13. Moyer JD and Henderson JF, Compartmentation of intracellular nucleotides in mammalian cells. CRC Crit Rev Biochem 19: 45-61, 1985.

14. Andersson M, Lewan L and Stenram U, Compartmentation of purine and pyrimidine nucleotides in animal cells. Int J Biochem 20: 1039-1050, 1988.

15. Fernandes DJ and Cranford SK, Dissociation of thymidylate biosynthesis from DNA biosynthesis by 5 fluoro-2'-deoxyuridine and 5,8-dideazaisofolic acid. Cancer Res 46: 1741-1747, 1986.

16. Danenberg KD and Danenberg PV, Activity of thymidylate synthetase and its inhibition by 5fluorouracil in highly enzyme-overproducing cells resistant to 10-propargyl-5,8-dideazafolate. Mol Pharmacol 36: 219-223, 1989.

17. Berger SH and Berger FG, Thymidylate synthase as a determinant of 5-fluoro-2'-deoxyuridine response in human colonic tumor cell lines. Mol Pharmacol 34: 474-479, 1988.

18. Berger SH, Barbour $\mathrm{KW}$ and Berger FG, A naturally occurring variation in thymidylate synthase structure is associated with a reduced response to 5-fluoro- $2^{\prime}$ deoxyuridine in a human colon tumor cell line. $\mathrm{Mol}$ Pharmacol 34: 480-484, 1988.

19. Garrett C, Wataya Y and Santi DV, Thymidylate synthetase. Catalysis of dehalogenation of 5-bromoand 5-iodo-2'-deoxyuridylate. Biochemistry 18: 27982804, 1979. 
20. Benson AB III, Trump DL, Cummings KB and Fischer $\mathrm{PH}$, Modulation of 5-iodo-2'-deoxyuridine metabolism and cytotoxicity in human bladder cancer cells by fluoropyrimidines. Biochem Pharmacol 34: 3925-3931, 1985.

21. Greene RF and Collins JM, Effects of leucovorin on idoxuridine cytotoxicity and DNA incorporation. Cancer Res 50: 6652-6656, 1990.

22. Vazquez-Padua MA, Risueno $\mathrm{C}$ and Fischer PH, Regulation of the activation of fluorodeoxyuridine by substrate competition and feedback inhibition in $647 \mathrm{~V}$ cells. Cancer Res 49: 618-624, 1989. 